\title{
C4d at Crossroads Between Post-Infectious Glomerulonephritis and C3 Glomerulopathy
}

This article was published in the following Dove Press journal:

International Journal of Nephrology and Renovascular Disease

\author{
Shaarif Bashir (D)' \\ Mudassar Hussain' \\ Aurangzeb Afzal ${ }^{2}$ \\ Usman Hassan' \\ Maryam Hameed' \\ Sajid Mushtaq'
}

'Department of Pathology, Shaukat Khanum Memorial Cancer Hospital and Research Centre, Lahore, 54000, Pakistan; ${ }^{2}$ Department of Nephrology, Lahore General Hospital, Lahore, 54000, Pakistan
Correspondence: Shaarif Bashir Department of Pathology, Shaukat Khanum Memorial Cancer Hospital and Research Centre, Lahore, Pakistan $\mathrm{Tel}+923334245144$

Email shaarifbashir@yahoo.com
Background: Post-infectious glomerulonephritis (PIGN) (immune complex-mediated glomerulonephritis) and C3 glomerulopathy are sub-types of glomerulonephritis (GN) with hypercellularity. Both have overlapping clinical and morphologic features on a kidney biopsy, however, the treatment and prognosis of these diseases are quite different making their distinction of utmost importance. Immune complex-mediated glomerulonephritis arises from glomerular deposition of immune-complexes (Igs) and C3 as a result of activation of classical (CP) and lectin pathways (LP). C4d is produced as a result of activation of the $\mathrm{CP} /$ LP. On the other hand, $\mathrm{C} 3$ glomerulopathy results from activation of alternative pathway of complement.

Aim: To distinguish between PIGN and C3 glomerulopathy with the help of C4d IHC stain. Materials and Methods: We studied 28 biopsies reported as GN with hypercellularity from January 2015 to January 2020. Clinical information, histological features and immunofluorescence patterns were analyzed. C4d IHC was performed on all the biopsies. Six known cases of immune complex-mediated GN were selected to act as a positive control for $\mathrm{C} 4 \mathrm{~d}$ staining.

Results: Amongst 28 cases originally reported as GN with hypercellularity, 18 were labeled as post-infectious $\mathrm{GN}$ and 10 as $\mathrm{C} 3$ glomerulopathy based on clinical information and serological findings. 13 of $18(72.2 \%)$ cases of PIGN had mild to moderate $(1-2+) \mathrm{C} 4 \mathrm{~d}$ staining, $2(11.1 \%)$ had strong (3+) staining and $3(16.7 \%)$ cases were negative for $\mathrm{C} 4 \mathrm{~d}$ staining. In the 10 biopsies of $\mathrm{C} 3$ glomerulopathy, mild (1+) $\mathrm{C} 4 \mathrm{~d}$ staining was noted only in $3(30 \%)$ biopsies. C4d had moderate to strong (2-3+) staining in the control group.

Conclusion: $\mathrm{C} 4 \mathrm{~d}$ IHC stain can be helpful in distinguishing PIGN from C3 glomerulopathy.

Keywords: proliferative, post-infectious, C3 glomerulopathy, C4d

\section{Plain Language Summary}

What is already known about this subject: There is a significant overlap between clinical, histological and IF findings of post-infectious glomerulonephritis (PIGN) and C3 glomerulopathy which are both types of glomerulonephritis (GN) with hypercellularity. The treatment plan for both entities is very different and that makes their differentiation from each other very important.

What this study adds: This study provides a method to differentiate PIGN from $\mathrm{C} 3$ glomerulopathy by the use of C4d IHC stain which is commonly available in centres all over the world.

What impact this may have on practice or policy: Definite sub-typing of GN with hypercellularity into PIGN and C3 glomerulopathy will massively help both the renal pathologists in rendering a definite diagnosis on tissue biopsy and the nephrologists in planning an appropriate treatment for the patient. 


\section{Introduction}

Glomerulonephritis with hypercellularity (previously known as Proliferative glomerulonephritis) is usually classified into immune complex mediated glomerulonephritis and complement-mediated glomerulonephritis (Mayo classification of membranoproliferative GN). ${ }^{1-3}$ This classification is based on pathophysiology along with light microscopic and immunofluorescence findings. Infection related GN is a type of immune complex mediated glomerulonephritis caused by glomerular deposition of immunoglobulins as a result of streptococcal infections, autoimmune diseases or hepatitis $B$ infection and occurs as a result of activation of the classical (CP) or lectin pathway (LP) of complement (Figure 1). Post-infectious GN (also known as poststreptococcal GN) is an important sub-type of infection related GN. The complement-mediated glomerulonephritis, also called C3 glomerulopathy, occurs by glomerular deposition of complementary factors produced from activation of the alternative pathway (AP) of the complement. $^{4-7} \mathrm{C} 3$ glomerulopathy includes both $\mathrm{C} 3$ glomerulopathy and dense deposit disease (DDD) which can only be differentiated by electron microscopy. $^{7}$

Immunofluorescence plays a pivotal part in the Mayo classification. Immune complex-mediated glomerulonephritis shows immunoglobulins on immunofluorescence, coupled with $\mathrm{C} 3$, due to activation of the $\mathrm{CP}$ by the immune complexes or LP by the microbial surfaces. On the other hand, in complement mediated glomerulonephritis ( $\mathrm{C} 3$ glomerulopathy), there is bright staining for $\mathrm{C} 3$, while immunoglobulins are typically negative. However, a small proportion of $\mathrm{C} 3$ glomerulopathy cases may show weak positivity for immunoglobulins on immunofluorescence. ${ }^{8,9}$ Consequently, C3 glomerulopathy is now defined as dominant C3 staining; at least two times the magnitude of any other immune reactants. ${ }^{8}$

\section{Pathogenesis}

Binding of $\mathrm{Clq}$ to the immune complexes activates the classic pathway resulting in activation of $\mathrm{C} 4$ and generation of $\mathrm{C} 4$ convertase. Therefore, binding of $\mathrm{C} 1 \mathrm{q}$ to $\mathrm{IgG} / \mathrm{IgM}$ is an initial event in the activation of the CP. C4d is a split product of $\mathrm{C} 4$ activation and points to the activation of CP. Activation

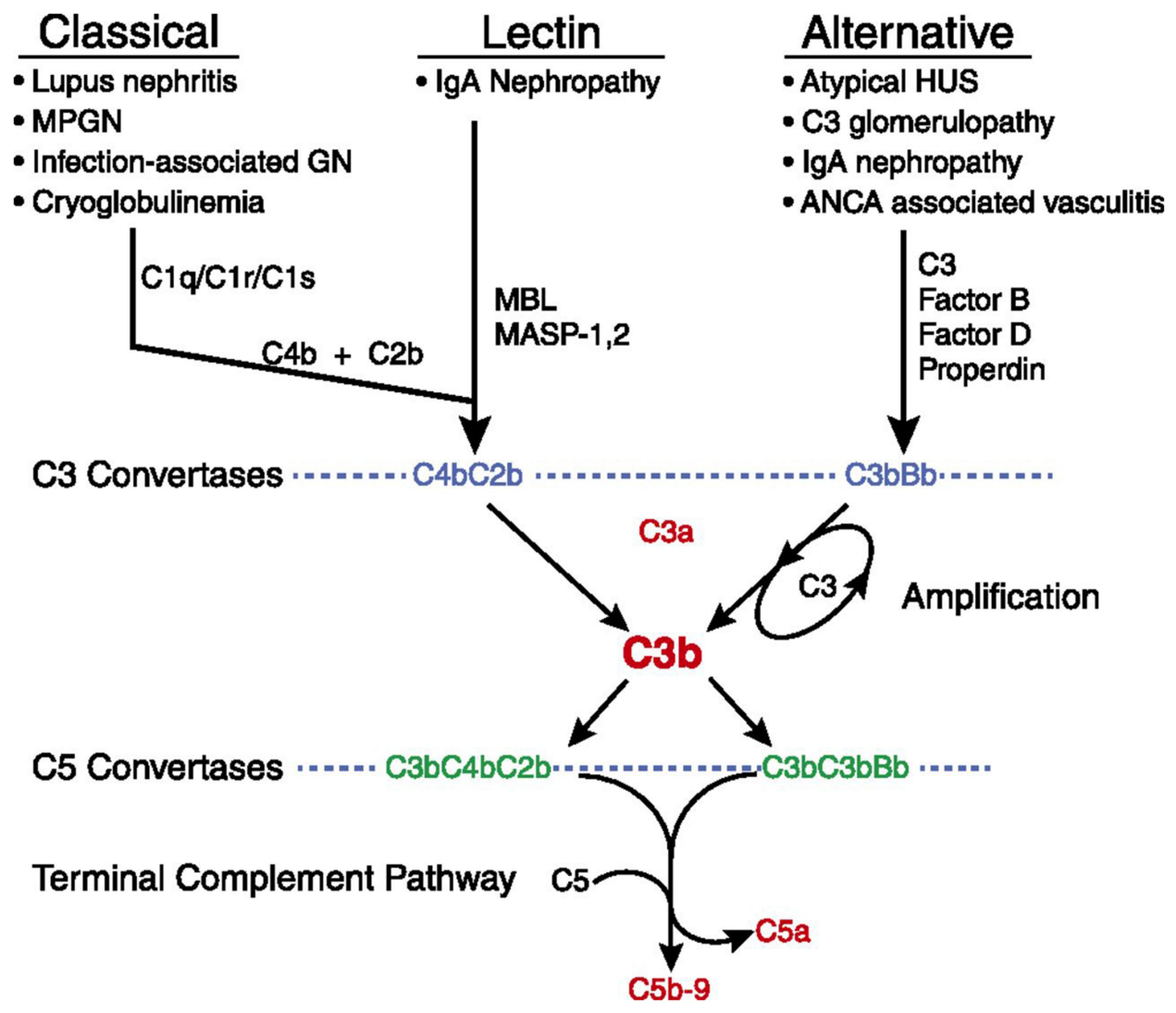

Figure I Overview of complement activation pathways. 
A

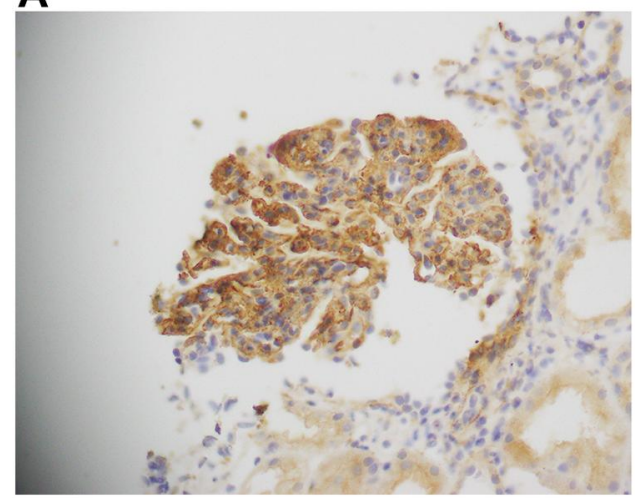

B

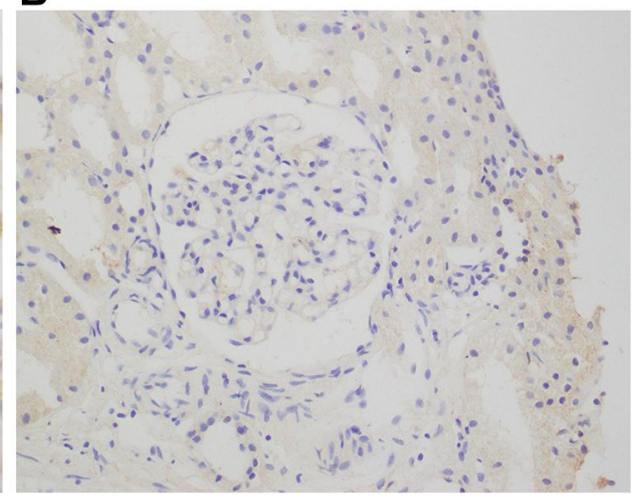

Figure 2 (A) Strong 3+ positivity of C4d IHC stain in a case of membranous GN (positive control group). (B) Negative C4d IHC staining in a case of minimal change disease (negative control group).

of $\mathrm{C} 4$ can also occur through the lectin pathway (LP) (Figure 1). In LP, mannose binding lectins bind to bacterial carbohydrate moieties and activate $\mathrm{C} 4$, causing production of $\mathrm{C} 3$ convertase. $^{10-13,22}$ So, C4d is also a by-product of LP activation. Another important point to note here is that $\mathrm{C} 4 \mathrm{~d}$ is commonly used as a marker of acute/chronic antibodymediated transplant rejection, ${ }^{14,15}$ but studies have shown that it is also positive in immune complex mediated glomerulopathies (Figure 2) like post-infectious GN (PIGN), lupus nephritis and membranous nephropathy, etc. ${ }^{16-22}$

Based on this, we believe that glomerular positivity of $\mathrm{C} 4 \mathrm{~d}$ can be used as a sign for post-infectious GN which is an immune mediated GN and occurs by activation of $\mathrm{CP} /$ LP. Conversely, negative glomerular $\mathrm{C} 4 \mathrm{~d}$ can be considered as a marker for C3 glomerulopathy because it does not involve CP/LP and only the alternative pathway is activated in which $\mathrm{C} 4 \mathrm{~d}$ is not generated. Furthermore, we believe that the trace/scant immunoglobulin staining may be seen in some patients of $\mathrm{C} 3$ glomerulopathy which may represent a small subset of patients that have activation of $\mathrm{CP} / \mathrm{LP}$ and thus weak C4d staining can be present in them.

\section{Materials and Methods}

28 biopsies reported as GN with hypercellularity (with a differential diagnosis of post-infectious GN and C3 glomerulopathy) from January 2015 to January 2020 at our centre were retrieved and reviewed by a panel of renal pathologists. For the waiver of informed consent, approval from institutional review board of Shaukat Khanum Memorial Cancer Hospital and Research Centre, Lahore, Pakistan was obtained prior to commencement of this study. Waiver of informed consent was obtained as most of the information was obtained from electronic medical records and there was no direct involvement of the study subjects. In compliance with the Declaration of Helsinki, patient information was recorded in an anonymous manner and without any identifiers to maintain confidentiality of data.

Pertinent clinical information including demographic features, serum C3, C4, ANA and ASO titers, history of fever, hematuria and sore throat in last three months was retrieved from electronic medical records (Hospital Information System) and via telephone calls.

Slides of formalin-fixed paraffin-embedded renal biopsies (maximum thickness 3-4 microns) stained with hematoxylin and eosin (H\&E) and special stains like Periodic Acid-Schiff (PAS), Masson's Trichrome and Jones methenamine silver (JMS) were used for the light microscopy. These three special stains (JMS, PAS and Trichrome) of kit company Roche Ventana were performed using Ventana's Benchmark Special stainer. The immunohistochemical stain (IHC) C4d was performed using Leica Bond III IHC Stainer with a retrieval time of 20 minutes. $\mathrm{C} 4 \mathrm{~d}$ staining in glomeruli was interpreted by the team of renal pathologists individually and the mean was documented. Furthermore, immunofluorescence (IF) results were also reviewed using the departmental pictorial database.

Both C4d glomerular positivity and IF (C3 and IgG) were scored from 0 to $3+$ based on intensity:

- Score 0: Negative.

- Score 1+: Weak staining.

- Score 2+: Moderate staining.

- Score 3+: Strong/Bright staining. 
Moreover, to act as positive control for C4d staining, 6 biopsies of immune complex mediated GN (3 of lupus nephritis and 3 of membranous GN) were also retrieved, reviewed and $\mathrm{C} 4 \mathrm{~d}$ IHC was performed on them. 6 biopsies of minimal change disease (MCD) and focal segmental glomerulosclerosis (FSGS) were used as negative control for C4d.

\section{Inclusion and Exclusion Criteria}

Biopsies with complete clinical information, ANA and ASO titer results and adequate light microscopy, IHC and IF information for review were included in the study. The biopsies with incomplete clinical history, lab results or inadequate material for light microscopy, IHC or IF studies were excluded.

\section{Results}

Of the total 28 biopsies, $17(60.7 \%)$ were of male and 11 $(39.3 \%)$ were of female patients with a mean age of $25.3 \pm$ 12 years. $26(92.9 \%)$ cases had a history of hematuria and $3+\mathrm{C} 3$ staining was noted in $24(85.7 \%)$ biopsies.

18 of $28(64.3 \%)$ biopsies had a history of sore throat and 17 amongst those 18 also had fever and raised ASO titer. They were labeled as PIGN. The remaining 10 $(35.7 \%)$ biopsies (with no history of sore throat or raised ASO titer) were designated as $\mathrm{C} 3$ glomerulopathy.

In the 18 PIGN biopsies, 11 (61.1\%) were males and 7 (38.9\%) were females with a mean age of 23.8 years. 17 patients $(94.4 \%)$ had raised ASO titer and a history of fever. C3, C4 levels were available for 9 patients, 4 of those $9(4.4 \%)$ had low C3 levels. C4 levels were normal in all $9(100 \%)$ patients. Four $(22.2 \%)$ were ANA positive. 5 of 18 biopsies $(27.7 \%)$ had negative $\mathrm{IgG}$ results, 8 $(44.4 \%)$ had $1+\operatorname{IgG}, 3$ (16.7\%) had $2+$ and $2(11.1 \%)$ had 3+ IgG staining. For C4d staining, 13 (72.2\%) had mild to moderate (1-2+) staining, $2(11.1 \%)$ had strong (3 + ) staining while $3(16.7 \%)$ biopsies were negative for $\mathrm{C} 4 \mathrm{~d}$ staining (same biopsies that were negative for $\mathrm{IgG}$ ) (Figure 3).

In the 10 biopsies of $\mathrm{C} 3$ glomerulopathy, 6 (60\%) were male and $4(40 \%)$ were females with a mean age of 28 years. None of the patients had a history of sore throat or raised ASO titer. C3, C4 levels were available for 4 patients, 3 of those 4 (75\%) had low C3 levels. C4 levels were normal in all $4(100 \%)$ patients. $2(20 \%)$ patients had a history of fever and were ANA positive as well. Mild (1+) IgG staining was observed in $3(30 \%)$ biopsies and mild (1+) C4d staining was also noted in the same $3(30 \%)$ biopsies. Interestingly, ANA was positive in two of those three $\mathrm{C} 4 \mathrm{~d} 1+$ biopsies (Table 1).

$\mathrm{C} 1 \mathrm{q}, \mathrm{IgA}$ and IgM by IF were negative in all the cases (Table 1). All 6 control biopsies showed strong 2-3+ staining for $\mathrm{C} 4 \mathrm{~d}$ IHC stain. (Figure 2A). All 6 biopsies of MCD and FSGS were negative for C4d (Figure 2B).

\section{Discussion}

In the last few decades, renal pathology has undergone remarkable advances due to widespread use of techniques like immunofluorescence studies and electron microscopy. This has led to the introduction of various new entities but has also posed new challenges to nephrologists and nephropathologists due to overlapping features between some of them. GN with hypercellularity is one such entity which encompasses both PIGN and C3 glomerulopathy.

PIGN (aka PSGN) arises as a result of streptococcal infection, usually with nephritogenic strains of group A beta-hemolytic streptococci, usually about three weeks after an episode of infection of pharynx or skin. It presents with hematuria, oliguria, edema and hypertension. There is activation of $\mathrm{CP} / \mathrm{LP}$ with raised ASO titer. $\mathrm{C} 4 \mathrm{~d}$ is a byproduct of activation of the CP/LP.

On the other hand, C3 glomerulopathy is a recently described entity which includes both the dense deposit disease (DDD) and C3 GN. ${ }^{22}$ It was first defined in a first C3 glomerulopathy meeting held in August 2012. ${ }^{7}$ Both acquired and genetic complement abnormities, especially mutations in CFH and CFI genes, are thought to be the causative factors. ${ }^{23}$ It also presents with hematuria, oliguria and proteinuria. There is activation of alternative pathway (AP) of complement and deposition of $\mathrm{C} 3$ and other complement fragments with minimal or no deposition of immune complexes/Igs. ${ }^{10-12,22}$

It was felt that there is significant overlap in the features of PIGN and $\mathrm{C} 3 \mathrm{GN}$, as both can present with proteinuria and hematuria and can have high C3 levels. On light microscopy, both share common histological features like glomerular enlargement and endocapillary proliferation, mesangial proliferation, focal wire loop deposits and active interstitial inflammation with mild patchy scarring in some cases (Figure 3). Both have bright C3 staining with or without IgG staining on IF and sub-epithelial deposits on electron microscopy. ${ }^{21}$ Clinical information, like history of infection/sore throat and ASO titer, can be of help in distinguishing the two but apart from that, there is a lot of overlap between the two entities and their differentiation on 
A

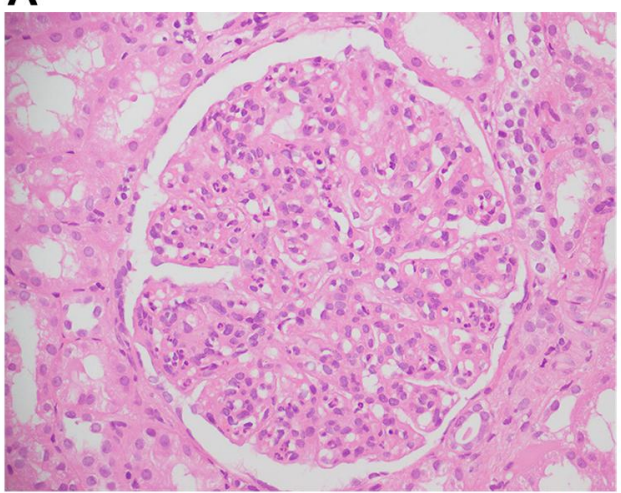

C

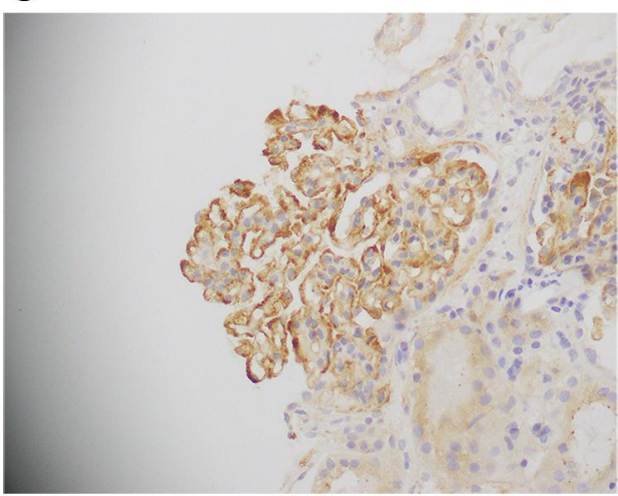

E

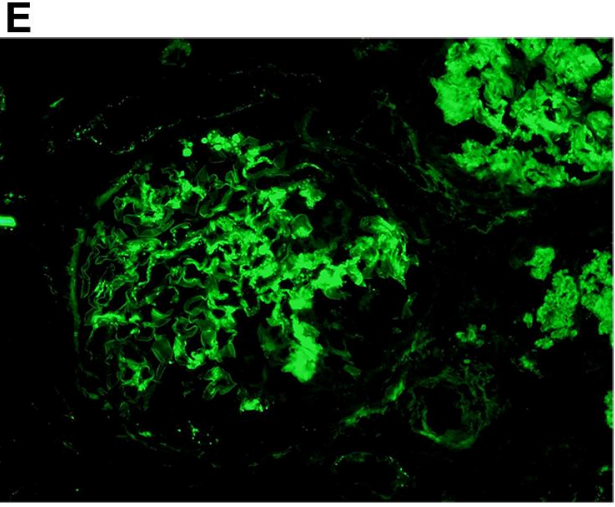

B

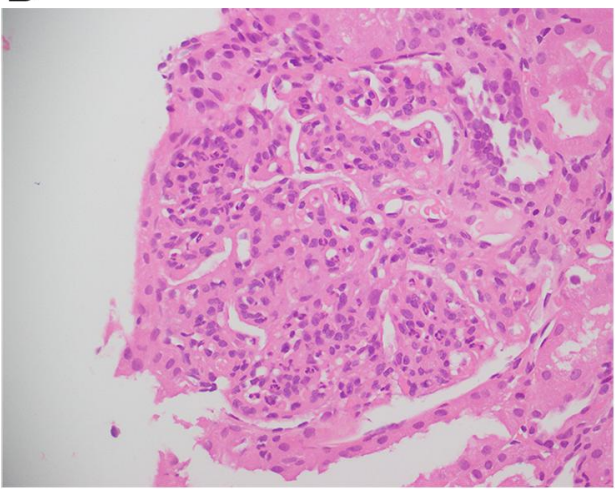

D

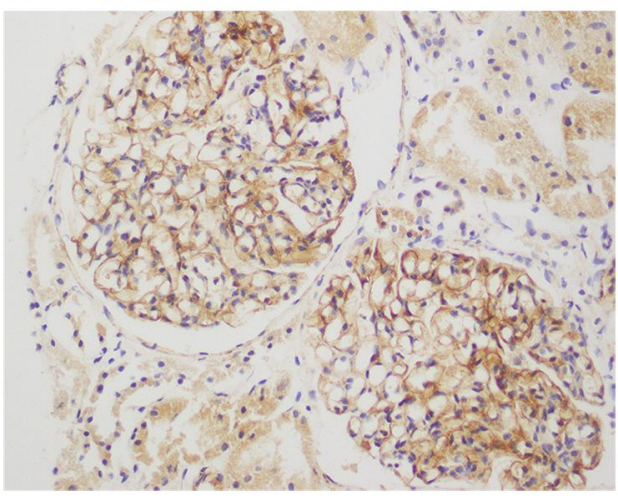

F

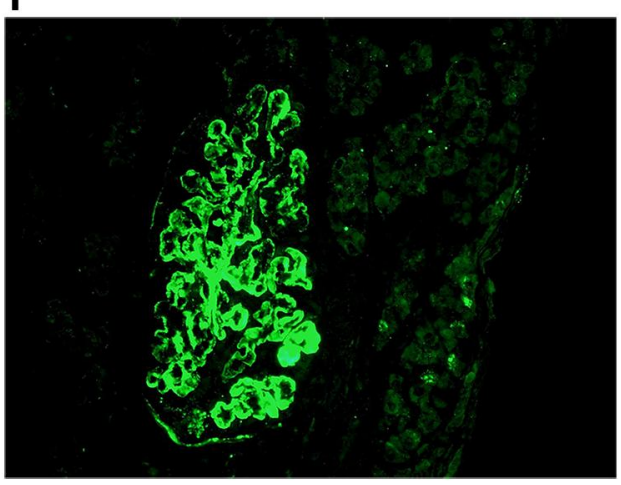

Figure 3 Post-infectious GN. (A and B) Periodic acid-Schiff stain showing glomerular enlargement, hypercellularity, endocapillary proliferation, mesangial proliferation and focal wire loop deposits (40x). (C and D) C4d IHC stain showing moderate 2+ staining (40x), (E) Immunofluorescence showing $2+$ granular staining for IgG, (F) Immunofluorescence showing bright 3+ granular staining for C3.

both clinical and histopathological grounds has remained a challenge for both the clinicians and pathologists.

From a clinical perspective, one may ask, why is it so important to differentiate the PIGN from C3 glomerulopathy? The fact is, it is of great clinical importance due to the following reasons:

Firstly, the treatment approach is different for both entities. In most cases of PIGN, antibiotics are the drug of choice and role of immunosuppression is controversial and is only reserved for severe cases. ${ }^{24}$ While in C3 glomerulopathy, use of immunosuppressive drugs like mycophenolate mofetil (MMF), along with steroids, is recommended. ${ }^{25}$ Previously, it was proposed to use the term 'glomerulonephritis with dominant C3' for such cases and let the clinical course of disease define the exact mechanism behind it. If it resolved over a period of a few weeks or months with the use of antibiotics alone, it was labeled as PIGN and if not, C3 glomerulopathy was suspected and immunosuppressive therapy was started. This approach of "wait and watch" has received criticism 


\begin{tabular}{|c|c|c|c|c|c|c|c|c|c|c|c|c|c|c|c|c|c|c|c|c|c|c|c|}
\hline$\frac{\sigma}{v}$ & 0 & 0 & 0 & 0 & 0 & 0 & 0 & 0 & 0 & 0 & 0 & 0 & 0 & 0 & 0 & 0 & 0 & 0 & 0 & 0 & 0 & 0 & 0 \\
\hline$\sum_{\underline{\underline{w 0}}}$ & 0 & 0 & 0 & 0 & 0 & 0 & 0 & 0 & 0 & 0 & 0 & 0 & 0 & 0 & 0 & 0 & 0 & 0 & 0 & 0 & 0 & 0 & 0 \\
\hline 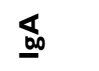 & 0 & 0 & 0 & 0 & 0 & 0 & 0 & 0 & 0 & 0 & 0 & 0 & 0 & 0 & 0 & 0 & 0 & 0 & 0 & 0 & 0 & 0 & 0 \\
\hline 导 & \pm & 0 & \pm & 0 & \pm & 0 & 0 & 0 & 0 & 0 & 0 & $\stackrel{+}{\sim}$ & \pm & $\stackrel{+}{\sim}$ & 0 & $\stackrel{+}{\sim}$ & 0 & $\stackrel{+}{m}$ & $\stackrel{+}{\sim}$ & $\stackrel{+}{\sim}$ & $\stackrel{+}{\sim}$ & $\stackrel{+}{\sim}$ & $\stackrel{+}{\sim}$ \\
\hline 比 昏 & \pm & 0 & \pm & 0 & \pm & 0 & 0 & 0 & 0 & 0 & 0 & \pm & 0 & \pm & 0 & $\stackrel{+}{\sim}$ & 0 & $\stackrel{+}{m}$ & \pm & \pm & \pm & $\stackrel{+}{\sim}$ & $\stackrel{+}{m}$ \\
\hline ט & $\stackrel{+}{m}$ & m & $\stackrel{+}{m}$ & $\stackrel{+}{m}$ & $\stackrel{+}{m}$ & $\stackrel{+}{m}$ & $\stackrel{+}{m}$ & $\stackrel{+}{m}$ & $\stackrel{+}{m}$ & $\stackrel{+}{m}$ & $\stackrel{+}{m}$ & $\stackrel{+}{m}$ & $\stackrel{+}{\sim}$ & $\stackrel{+}{m}$ & $\stackrel{+}{m}$ & $\stackrel{+}{m}$ & $\stackrel{+}{\sim}$ & $\stackrel{+}{m}$ & $\stackrel{+}{m}$ & $\stackrel{+}{m}$ & $\stackrel{+}{m}$ & $\stackrel{+}{m}$ & $\stackrel{+}{\sim}$ \\
\hline 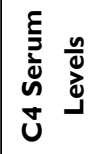 & 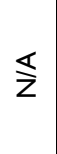 & 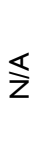 & $\$$ & $\begin{array}{l}\overline{\tilde{g}} \\
\text { o } \\
\text { z }\end{array}$ & $\begin{array}{l}\overline{\widetilde{g}} \\
\bar{\xi} \\
\text { z }\end{array}$ & 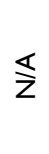 & 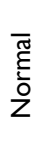 & $\$$ & $\overleftrightarrow{s}$ & $\begin{array}{l}\overline{\widetilde{\Xi}} \\
\text { है } \\
\text { Z }\end{array}$ & $\begin{array}{l}\overline{\tilde{g}} \\
\overline{\mathrm{g}} \\
\mathrm{z}\end{array}$ & $\begin{array}{l}\overline{\tilde{g}} \\
\bar{\xi} \\
\bar{z}\end{array}$ & $\$$ & $\begin{array}{l}\overline{\tilde{g}} \\
\bar{\xi} \\
\text { Z }\end{array}$ & $\overleftarrow{z}$ & $\overleftarrow{z}$ & 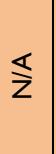 & $\begin{array}{l}\overline{\mathrm{g}} \\
\overline{\mathrm{E}} \\
\overline{\mathrm{z}}\end{array}$ & $\overleftarrow{z}$ & $\begin{array}{l}\overline{\widetilde{J}} \\
\text { ò } \\
\text { Z }\end{array}$ & 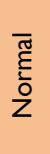 & $\overleftrightarrow{s}$ & $\begin{array}{l}\overline{\widetilde{\sigma}} \\
\overline{\mathrm{g}} \\
\mathrm{Z}\end{array}$ \\
\hline
\end{tabular}

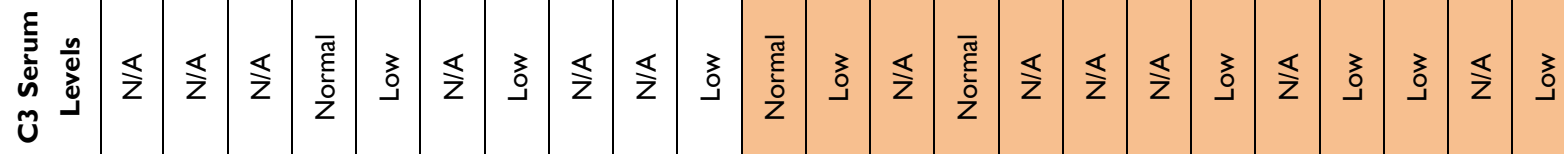

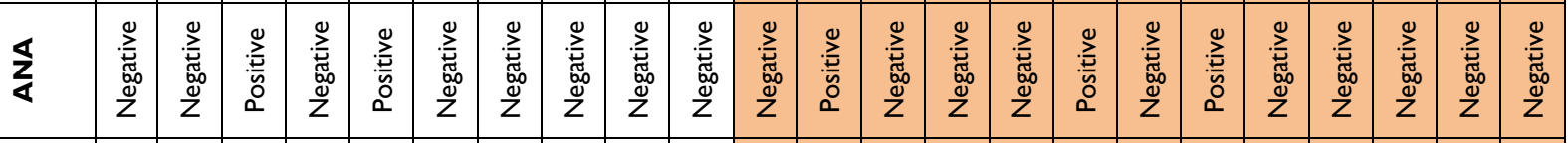

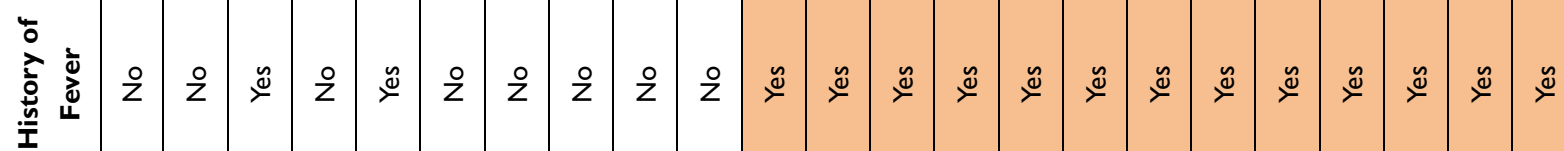

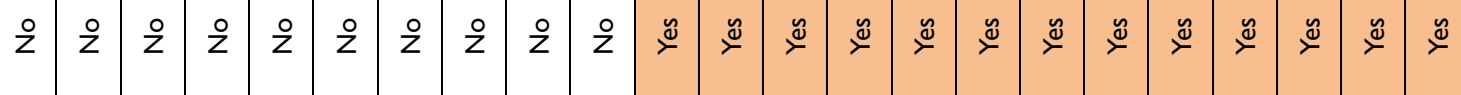

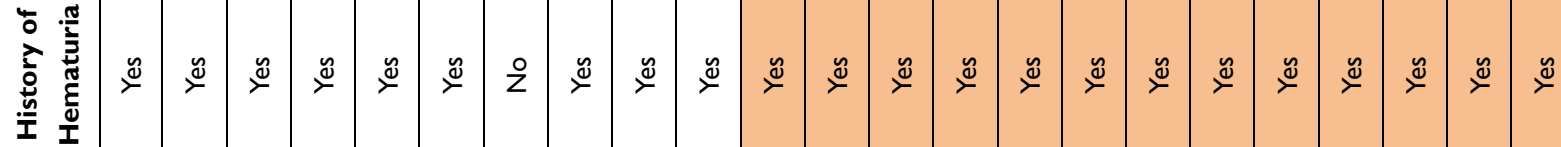

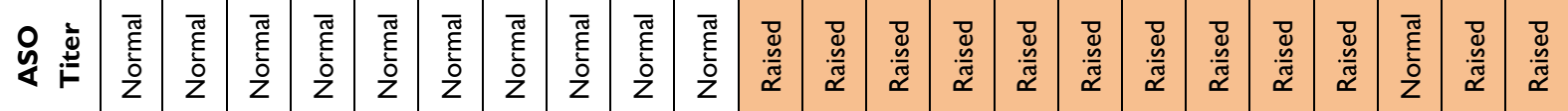

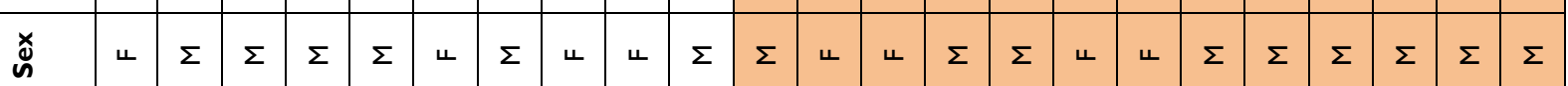

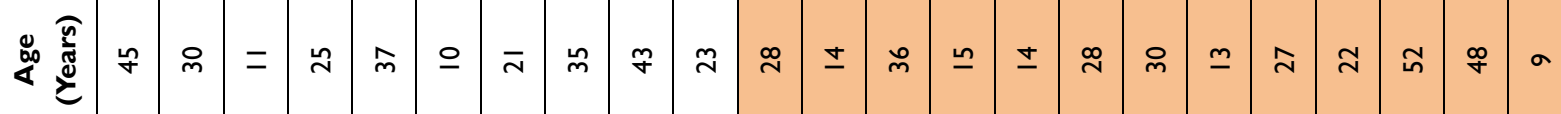
$\frac{\widehat{a ̀ n}}{\frac{0}{0}} \frac{0}{2}$

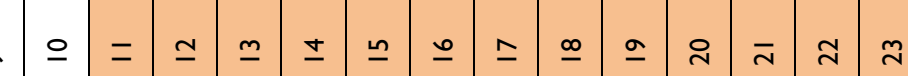




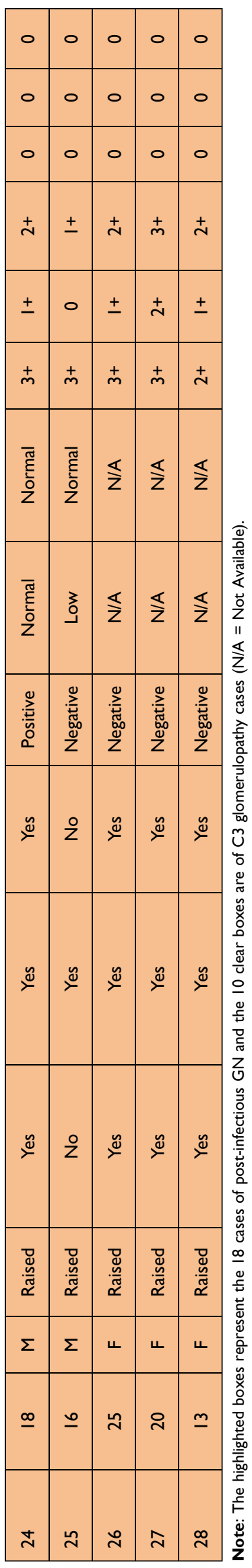

as precious time is lost and it leaves the patient without a definite diagnosis posing challenges on his/hers physical and mental health. Some may argue that why do we not start antibiotics and immunosuppressives together? Although it seems logical we have to keep in mind that immunosuppressive drugs like MMF have several side effects (vomiting, headaches, bone marrow suppression, depression, teratogenicity and risk of infection) ${ }^{26,27}$ and they massively increase the treatment costs. This is especially important for under-developed countries, where resources and health facilities are limited.

Secondly, from a prognostic point of view, PIGN has an excellent prognosis especially in children and adults but in elderly, the prognosis is relatively poor both in terms of dialysis dependency and mortality. ${ }^{24}$ On the other hand, C3 GN is a relatively newly described entity and the longterm prognosis is not well documented, however early intervention has shown a better prognosis. But early intervention is only possible if a definitive diagnosis is made. ${ }^{25}$

Firstly, we performed $\mathrm{C} 4 \mathrm{~d}$ IHC on six biopsies of immune-complex mediated GN so that these cases can be used as a positive control. Three of the biopsies were of membranous GN and the other three of lupus nephritis, both diagnosed on microscopy and immunofluorescence. C4d was strong $(2-3+)$ positive in all $6(100 \%)$ cases, indicating the activated CP/LP pathway (Figure 2).

After establishing a positive control, we chose 28 biopsies reported as GN with hypercellularity. Most of them were $\mathrm{C} 33+$ on IF and some were IgG positive as well. Relevant information like history of sore throat, fever and ASO titer was retrieved. 18 of 26 patients had a history of sore throat and all 18 of them had raised ASO titer as well (Table 1). They were sub-categorized as post-infectious GN. The remaining 10 biopsies were labeled as C3 glomerulopathy. C4d was performed and results were interpreted independently by a three member team of renal pathologists. The results were then compared and an average score was calculated for each biopsy.

\section{C4d in Post-Infectious GN}

Among the 18 biopsies of post-infectious GN, 13 (72.2\%) had mild to moderate $(1-2+)$ staining for $\mathrm{C} 4 \mathrm{~d}$ and 2 $(11.1 \%)$ had strong $(3+)$ staining suggesting activation of the $\mathrm{CP} / \mathrm{LP}$ as a result of microbial infection. $3(16.7 \%)$ biopsies were negative for $\mathrm{C} 4 \mathrm{~d}$ staining which might represent the activation of an atypical pathway in a subset of post-infectious GN (atypical post-infectious GN) (Figure 3). 


\section{C4d in C3 Glomerulopathy}

7 of $10(70 \%)$ biopsies of C3 glomerulopathy had a negative C4d staining while $3(30 \%)$ had mild (1+) staining of C4d. Amongst the three $\mathrm{C} 3$ glomerulopathy biopsies with mild (1+) C4d staining, two were ANA positive, suggesting a contribution by an autoimmune process in the background leading to activation of CP/LP of the complement and development of glomerulopathy; which on the basis of bright C3 positivity and mild (1+) IgG positivity was categorized as $\mathrm{C} 3$ glomerulopathy. Also, the three biopsies with mild (1+) $\mathrm{C} 4 \mathrm{~d}$ staining had $1+\operatorname{IgG}$ positivity while the rest of the biopsies were IgG negative.

In a similar study, Sethi et $\mathrm{al}^{22}$ performed $\mathrm{C} 4 \mathrm{~d}$ on $\mathrm{C} 3$ glomerulopathy and post-infectious GN biopsies. C4d was negative in 24 of $30(80 \%)$ of C3 glomerulopathy cases compared to 7 of $10(70 \%)$ in our study. In the postinfectious GN category, 6 of $13(46.2 \%)$ biopsies had $1-2+\mathrm{C} 4 \mathrm{~d}$ staining in their study compared to 13 of 18 $(72.2 \%)$ in ours. This indicates that $\mathrm{C} 4 \mathrm{~d}$ can be a very helpful diagnostic tool in distinguishing the two entities.

The limitations of our study included a relatively small sample size precluding adequate statistical analysis, the unavailability of serum protein electrophoresis, $\mathrm{CH} 50$ in plasma, electron microscopy and genetic analysis.

\section{Conclusion}

Use of C4d IHC stain is a quick and a very helpful method in distinguishing PIGN and C3 GN. It should be incorporated in the routine sign-out practice when dealing with a case of GN with hypercellularity.

\section{Acknowledgments}

The authors would like to acknowledge the lab staff at our centre, who work tirelessly to make sure that renal biopsies are processed according to the highest standards of quality.

\section{Author Contributions}

$\mathrm{S}$. Bashir searched the data for the article and designed the whole manuscript. M. Hussain, U. Hassan, and S. Mushtaq performed the histological review. S. Bashir, A. Afzal and M. Hameed contributed to the discussions of the article content and S. Bashir and M. Hussain did the final editing of the manuscript before submission. All authors contributed to data analysis, drafting or revising the article, have agreed on the journal to which the article will be submitted, gave final approval of the version to be published, and agree to be accountable for all aspects of the work.

\section{Disclosure}

The authors declare no conflicts of interests.

\section{References}

1. Sethi S, Fervenza FC. Membranoproliferative glomerulonephritis: pathogenetic heterogeneity and proposal for a new classification. Semin Nephrol. 2011;31:341-348.

2. Sethi S, Fervenza FC. Membranoproliferative glomerulonephritisa new look at an old entity. $N$ Engl J Med. 2012;366:1119-1131. doi:10.1056/NEJMra1108178

3. Sethi S. Etiology-based diagnostic approach to proliferative glomerulonephritis. Am J Kidney Dis. 2014;63:561-566. doi:10.1053/j.ajkd.2013.11.019

4. Servais A, Frémeaux-Bacchi V, Lequintrec M, et al. Primary glomerulonephritis with isolated $\mathrm{C} 3$ deposits: a new entity which shares common genetic risk factors with haemolytic uraemic syndrome. J Med Genet. 2007;44:193-199. doi:10.1136/jmg.2006.045328

5. Sethi S, Fervenza FC, Zhang Y, et al. Proliferative glomerulonephritis secondary to dysfunction of the alternative pathway of complement. Clin J Am Soc Nephrol. 2011;6:1009-1017. doi:10.2215/CJN.07110810

6. Sethi S, Fervenza FC, Zhang Y, et al. C3 glomerulonephritis: clinicopathological findings, complement abnormalities, glomerular proteomic profile, treatment, and follow-up. Kidney Int. 2012;82:465-473. doi:10.1038/ki.2012.212

7. Gale DP, de Jorge EG, Cook HT, et al. Identification of a mutation in complement factor H-related protein 5 in patients of Cypriot origin with glomerulonephritis. Lancet. 2010;376:794-801. doi:10.1016/ S0140-6736(10)60670-8

8. Pickering MC, D'Agati VD, Nester CM, et al. C3 glomerulopathy: consensus report. Kidney Int. 2013;84:1079-1089. doi:10.1038/ki.2013.377

9. Larsen CP, Walker PD. Redefining C3 glomerulopathy: 'C3 only' is a bridge too far. Kidney Int. 2013;83:331-332. doi:10.1038/ki.2012.385

10. Hou J, Markowitz GS, Bomback AS, et al. Toward a working definition of $\mathrm{C} 3$ glomerulopathy by immunofluorescence. Kidney Int. 2014;85:450-456. doi:10.1038/ki.2013.340

11. Espinosa M, Ortega R, Sánchez M, et al. Spanish Group for Study of Glomerular Diseases (GLOSEN): association of C4d deposition with clinical outcomes in IgA nephropathy. Clin J Am Soc Nephrol. 2014;9:897-904. doi:10.2215/CJN.09710913

12. Sethi S, Sullivan A, Smith RJ. C4 dense-deposit disease. $N$ Engl J Med. 2014;370:784-786. doi:10.1056/NEJMc1309449

13. Ohsawa I, Ohi H, Endo M, et al. Evidence of lectin complement pathway activation in poststreptococcal glomerulonephritis. Kidney Int. 1999;56:1158-1159. doi:10.1046/j.1523-1755.1999.00642.x

14. Collins AB, Schneeberger EE, Pascual MA, et al. Complement activation in acute humoral renal allograft rejection: diagnostic significance of C4d deposits in peritubular capillaries. J Am Soc Nephrol. 1999;10:2208-2214.

15. Cohen D, Colvin RB, Daha MR, et al. Pros and cons for C4d as a biomarker. Kidney Int. 2012;81:628-639. doi:10.1038/ki.2011.497

16. Batal I, Chalasani G, Wu C, et al. Deposition of complement product $\mathrm{C} 4 \mathrm{~d}$ in anti-glomerular basement membrane glomerulonephritis. $\mathrm{Am}$ J Kidney Dis. 2009;53:1098-1101. doi:10.1053/j.ajkd.2008.10.008

17. Batal I, Liang K, Bastacky S, et al. Prospective assessment of C4d deposits on circulating cells and renal tissues in lupus nephritis: a pilot study. Lupus. 2012;21:13-26. doi:10.1177/0961203311422093

18. Kusunoki Y, Itami N, Tochimaru H, et al. Glomerular deposition of C4 cleavage fragment $(\mathrm{C} 4 \mathrm{~d})$ and C4-binding protein in idiopathic membranous glomerulonephritis. Nephron. 1989;51:17-19. doi: $10.1159 / 000185234$ 
19. Rodriguez EF, Cosio FG, Nasr SH, et al. The pathology and clinical features of early recurrent membranous glomerulonephritis. Am J Transplant. 2012;12:1029-1038. doi:10.1111/j.1600-6143.2011.03903.x

20. Dhir V. Is cellular C4d a good biomarker for SLE nephritis? Lupus. 2012;21:1036. doi:10.1177/0961203312444496

21. Mazdak A, Wang S, Henriksen KJ, et al. Revisiting post-infectious glomerulonephritis in the emerging era of $\mathrm{C} 3$ glomerulopathy. Clin Kidney J. 2016;9(3):397-402. doi:10.1093/ckj/sfw032

22. Sethi S, Nasr SH, De Vriese AS, et al. C4d as a Diagnostic Tool in Proliferative GN. J Am Soc Nephrol. 2015;26(11):2852-2859. doi:10.1681/ASN.2014040406

23. Servais A, Noël LH, Roumenina LT, et al. Acquired and genetic complement abnormalities play a critical role in dense deposit disease and other C3 glomerulopathies. Kidney Int. 2012;82(4):454-464. doi:10.1038/ki.2012.63
24. Rodriguez-Iturbe B, Musser JM. Musser JM: the current state of poststreptococcal glomerulonephritis. J Am Soc Nephrol. 2008;19 (10):1855-1864. doi:10.1681/ASN.2008010092

25. Cook HT. C3 glomerulopathy. F1000Res. 2017;6:248. doi:10.12688/ f1000research.10364.1

26. Chaudhry V, Cornblath DR, Griffin JW, et al. Mycophenolate mofetil: a safe and promising immunosuppressant in neuromuscular diseases. Neurology. 2001;56(1):94-96. doi:10.1212/wnl.56.1.94

27. Draper HM. Depressive disorder associated with mycophenolate mofetil. Pharmacotherapy. 2008;28(1):136-139. doi:10.1592/ phco.28.1.136

\section{Publish your work in this journal}

The International Journal of Nephrology and Renovascular Disease is an international, peer-reviewed open-access journal focusing on the pathophysiology of the kidney and vascular supply. Epidemiology, screening, diagnosis, and treatment interventions are covered as well as basic

Submit your manuscript here: https://www.dovepress.com/international-journal-of-nephrology-and-renovascular-disease-journal science, biochemical and immunological studies. The manuscript management system is completely online and includes a very quick and fair peer-review system, which is all easy to use. Visit http://www.dovepress.com/testimonials.php to read real quotes from published authors. 